\title{
Face Lift Surgery for Reconstructed Virtual Humans
}

\author{
Rafael Pagés, Sergio Arnaldo and Francisco Morán \\ Grupo de Tratamiento de Imágenes \\ Universidad Politécnica de Madrid, Spain \\ rps@gti.ssr.upm.es
}

\begin{abstract}
We introduce an innovative, semi-automatic method to transform low resolution facial meshes into high definition ones, based on the tailoring of a generic, neutral human head model, designed by an artist, to fit the facial features of a specific person. To determine these facial features we need to select a set of "control points" (corners of eyes, lips, etc.) in at least two photographs of the subject's face. The neutral head mesh is then automatically reshaped according to the relation between the control points in the original subject's mesh through a set of transformation pyramids. The last step consists in merging both meshes and filling the gaps that appear in the previous process. This algorithm avoids the use of expensive and complicated technologies to obtain depth maps, which also need to be meshed later.
\end{abstract}

Keywords-Computer Graphics; Virtual Reality; Facial Animation.

\section{INTRODUCTION}

Meshes representing human bodies are among the hardest to model. Essential for a vast array of applications, such as special effects in the film industry, video-games, etc., these meshes are usually designed by hand by 3D artists. However, there exist methods for obtaining these meshes from real human bodies. One of these methods uses a set of photographs of the same subject taken simultaneously from different angles. In each of those images, the algorithm is able to extract the silhouette of the subject, and, if the calibration parameters of the cameras are known, it can extract a visual hull (VH), which can be thought of as a 3D silhouette defined as the intersection of the human-shaped cones. After voxelizing the VH, a 3D mesh can be extracted using the marching cubes algorithm.

The main problem of 3D meshes obtained from VHs is that concavities in the original body, such as the eye sockets, cannot be detected. Moreover, if the mesh undergoes a typical smoothing process, important information can get lost, especially in the face area. Because of this, we need algorithms that enhance all the distinctive face features by increasing polygonal density locally. These technologies usually detect the depth of a set of points that lie on a specific part of the surface with a higher accuracy. The set of points is triangulated and added to the mesh. This last step requires very complex and CPU-intensive algorithms and does not generate any kind of semantic information, which could be useful in case the face needs to be animated. To obtain these accurate 3D points on a human face, a 3D scanner (which could be based on structured light, for instance) is typically used. These scanners have the main advantage of producing 3D depth maps with big precision but introduce in the system many new complications as, for instance, calibration issues. Moreover, they are normally expensive, which could be a big drawback in less economically ambitious projects.

The goal of the process described in this paper is not just creating a facial 3D mesh but increasing the level of detail in the region of the mesh corresponding to the face in a low resolution mesh. For that, we transform a generic neutral facial mesh (which could have been created by an artist) using information about the facial features of the actual person. The algorithm suits best meshes which have a homogeneous triangle size throughout their surface. In this case, the level of detail for parts of the body such as the legs may be enough with the original triangle size, but that is not true for the face, which is the part of the body most sensitive to recognition by humans. Our algorithm is especially good in cases where additional devices would be too expensive to increase the resolution of a VH. Besides, thanks to the semi-automatic nature of the system, the set of control points could be used for 3D facial animation.

Figure 1 shows several partial results along the process, including the initial data. Our system consists in three main steps:

- In the first step, the important points of the face are selected both in the images and in the neutral head mesh, defining a set of "control points". Using these points, the area which will be replaced is defined and so are the "transformation pyramids" (TPs).

- Here is where the neutral face mesh is transformed using the coordinates obtained from the TPs. The unused areas of both the original and neutral meshes are deteled and, after that, they are merged using the topological information we have.

- As a result from the previous process, we have a mesh with important holes so, in the last step, these holes will be sewed, and the facial section will be completelly adapted to the original mesh.

\section{PREVIOUS WORK}

As virtual human models have been extensively used in many different applications, it is logical to think that finding an effective method to create 3D meshes representing human 

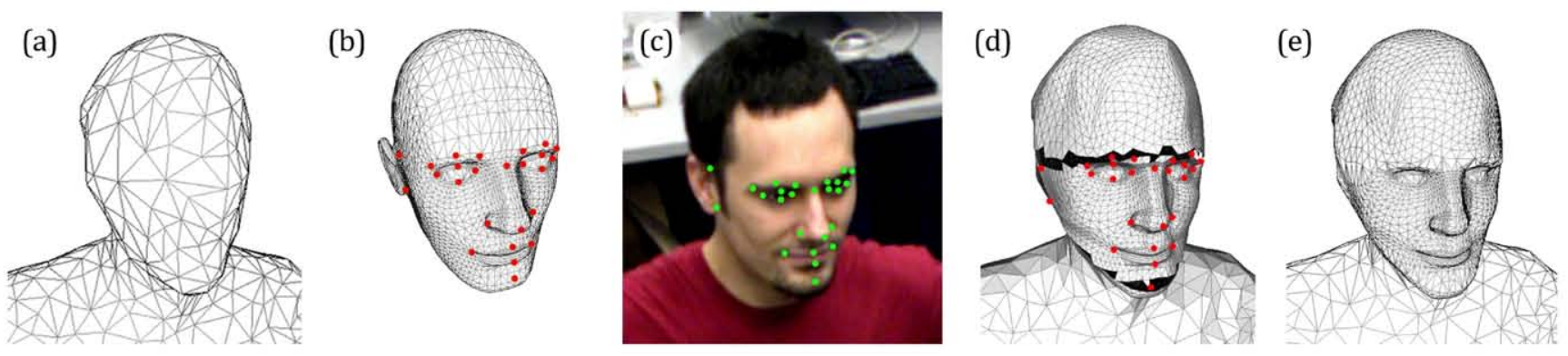

Figure 1. (a) Original low resolution mesh. (b) Neutral head mesh, with control points highlighted. (c) One of the photographs of the subject, with control points manually selected. (d) Resulting mesh after transformation. (e) Final mesh after sewing the holes.

bodies and particulary human faces is a very well studied issue. Actually, there exist different systems based on pattern recognition which, opposite to ours, use only one frontal image of the subject to recreate 3D facial animations as, for instance, Jiang's system [1], which uses a reconstruction approach for gesture recognition with variant pose, illumination and expression. Patel and Zaveri [2], apart from using just one image, model a neutral 3D mesh similarly to us but without merging it into a real 3D virtual human model, and with good results for animating the face, but not as good in terms of fidelity to the subject's face. Using two images, as we do, we have the work of Lee et al. [3] who use a semiautomatic method which detects facial features thanks to structured snakes, and allows them to animate the face. Nevertheless, the results are not very realistic, and the model looks more like a cartoon character. One of the algorithms with best results is the one proposed by Blanz and Vetter [4], who model a very realistic 3D facial model in two main steps: analyzing the subject face and modelling the 3D mesh. The main problem this algorithm has is that, for creating a very good model, a big and complete database of 3D facial models is needed to be able to reconstruct any sex or race; moreover, it calculates a complex cost function so it needs considerable computation resources.

\section{PRoposed TECHNIQUE}

\section{A. Initial steps}

To change the shape of the input mesh, a set of "control points" (CPs) will be used. In our case, we have used 28 CPs, highlighted in Figure 2, but this criterion is totally flexible if the pyramids are defined accordingly later. The CPs represent some of the most unambiguous points in the face, such as the tip of the nose or the corners of the mouth and eyes. The detection of these points is not discussed in this paper. In the example shown in Figure 2, they were manually selected in two of the photographs of the subject whose face was reconstructed. To obtain the 3D position of these CPs we use the 3D triangulation system proposed by Hartley [5], which guesses the position of a CP by calculating the 3D point $Q$ that minimizes the sum of the distances from its projections $\left(\operatorname{Proj}_{i}(Q)\right)$ to the guessed

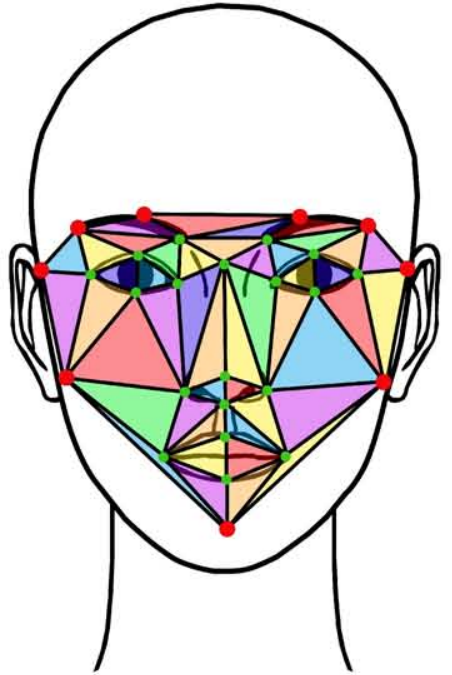

Figure 2. Set of CPs and bases of the TP used in the process. External CPs are highlighted in red.

projection $\left(\overrightarrow{q_{i}}\right)$ in each photograph. After that, they are also selected among the vertices of the neutral facial mesh. It is very important that the CPs be selected accordingly in both images and the mesh, because the relations between them will be key in the transformation.

After this, all the CPs are placed both in the original mesh and in the neutral one. The most external CPs will define what we call the "mask", which determines the areas which will be modified in both meshes. Specifically, in the original mesh, the triangles which reach this area will be removed so they can be substituted; on the other hand, in the neutral head mesh, the triangles inside the mask will be transformed, and the rest will be removed.

To be able to perform the transformation taking into account the facial features of the subject, we need to define a new structure. The mask volume is in turn defined by a set of "transformation pyramids" (TPs), all sharing the same apex, a point called "barycenter", situated approximately in the centre of the volume of the head and calculated from the position of some of the CPs. The base of the TP is a 
triangle defined by three CPs. Figure 2 shows the bases of all the control pyramids. It is important to note that these pyramids are defined by their bases but not bound by them: their volume is theoretically infinite.

\section{B. Transformation of the neutral head mesh}

Once we know the positions of the CPs, we treat them according to their type: the position of the internal ones is kept, while the position of the external ones is adjusted to the position of the closest point in the original mesh, so as to favor an eventual continuity between the mask and the original mesh. But before the positions of the external CPs are adjusted, the triangles of the original mesh are subdivided so that their resolution matches better that of the neutral head mesh. This will be beneficial in later steps of the algorithm, when both the artificial mask and the original mesh have to be sewn together. Also, the new position of the external points will be more accurate if the triangles of this part of the mesh are smaller, even if the detail of the face features is not yet present. The subdivision of the mesh can be done using Dyn's butterfly scheme [6]. We can save time by subdividing only the triangles in the head. To identify them, we can define a "guillotine" plane, by means of the (temporary) position of the lowest CP: the triangles corresponding to the head are those whose three vertices are above the guillotine plane.

The definitive position of all the CPs is known only after the positions of the external CPs have been updated to those of their closest points in the original subdivided mesh. It is important at this point to decide which triangles we want to transform: the neutral mesh might contain all the triangles needed to represent a whole head, but we only need the ones corresponding to the inner part of the external CPs, i.e., what we call the mask. In order to select the mask triangles, we need to define the mask volume. A triangle belongs to the mask if all its vertices belong to the mask volume. A vertex belongs to the mask volume if it belongs to one of the control pyramids. In this case, the transformation it undergoes depends only on the three CPs that define its pyramid (although, implicitly, it will also depend on the CPs the position of the barycenter depends on). Therefore, the transformation of the initial neutral head mesh can be interpreted as a set of transformations of the control pyramids: from the position of the CPs in the neutral mesh (totally accurate) to the position of the CPs in the original mesh (guessed by the algorithm).

In order to "attach" the position of a vertex to those of the points that define a pyramid, we create an auxiliary coordinate system. Let $P$ be the vertex we want to transform, $B$ the barycenter and $C P_{1}, C P_{2}$ and $C P_{3}$ the three CPs that define the base of the pyramid. Let $C$ be the intersection of the line that contains $P$ and $B$ with the plane that contains $C P_{1}, C P_{2}$ and $C P_{3}$. As illustrated by Figure 3, any position of $P$ inside the pyramid can be uniquely defined by the set

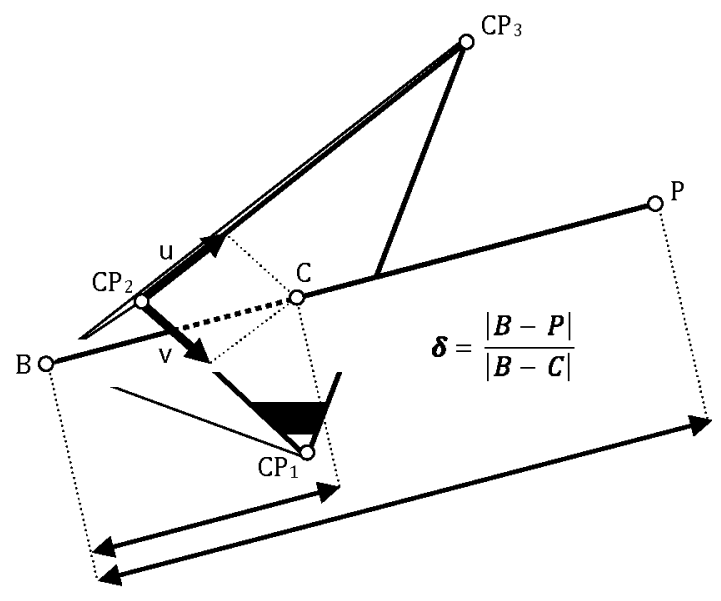

Figure 3. Example of a control pyramid and how the important parameters for the transformation are obtained.

$(u, v, \delta)$, where $u$ and $v$ are the coordinates of $C$ in the base defined by $C P_{2} C P_{1}$ and $C P_{2} C P_{3}$. It is possible to see the definition of $\delta$ also in Figure 3.

Thus, the control pyramids fulfil two goals: they provide a way to transform the mask points and they select the points we need to transform. Before performing any transformation, the triangles of the neutral mesh with at least one vertex outside the mask volume are deleted, so that only those belonging to the mesh remain. For the original mesh, the process will be the opposite: in this case, the triangles with at least one vertex inside the control pyramids are deleted, so that they can be replaced by the transformed synthetic ones.

\section{Filling the holes}

The output of the previous step is a human mesh with a detailed face but with a lot of holes around the mask: see Figure 1 (d). The only "bridges" between these two parts of the meshes may be the CPs, which may or may not be part of triangles in the mesh:

- A CP is contained in the mask if there is at least one triangle with its other two vertices inside the mask volume.

- $\mathrm{A} \mathrm{CP}$ is contained in the original mesh if it belongs to at least one triangle whose other two vertices do not belong to the transformed mask volume.

If a $\mathrm{CP}$ is contained in both the mask and the original mesh, it will not need to undergo the "bridging" process (see Figure 4). For all the rest, we will have to add one triangle per "shore" they are not connected to. The vertices that define a bridge triangle are the $\mathrm{CP}$ and those contained in the closest edge to the $\mathrm{CP}$ in the corresponding shore.

The result of the bridging process is a mesh with a set of holes (as many as external CPs) which we need to fill. Some of these gaps, such as those above the eyes, are relatively 

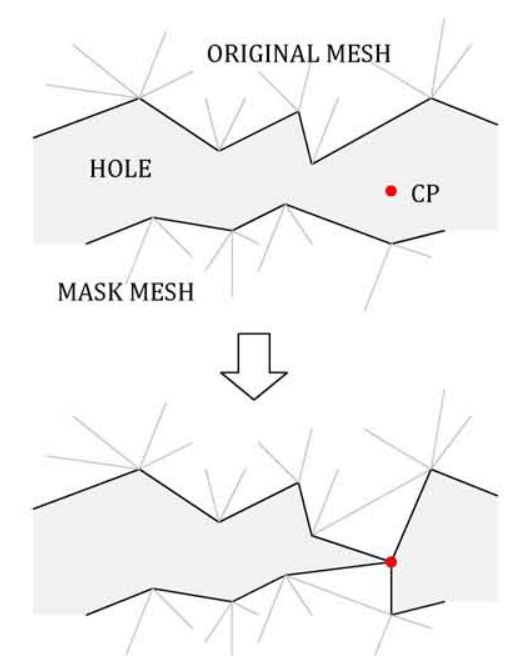

Figure 4. A bridge is created to join both the original and the mask meshes.

small and easy to fill; this is because there is a higher density of CPs in those mesh regions. However, there are others, such as the ones between the ears and the chin, which are quite bigger and not only harder to fill in a topological way, but also in a geometrical way: there is usually a "step" between both shores in the normal direction to the mesh surface. This forces us to modify the position of the vertices of one shore to adapt them to that of the other. Because we believe the original mesh resembles the human body more closely in parts which are further away from the CPs, we modify the vertices on the shore of the transformed mask.

For each hole, we make a list with the edges belonging to the original mesh and another with those of the mask. After that, we travel through the edges in the mask searching in each case for its closest edge in the original mesh. Once it is found, we move the two vertices of that edge to the position where the closest corresponding edge vertices are. We take great care in avoiding triangle intersections, and also deleting repeated vertices and edges after the process.

The final step is to triangulate the final holes. Luckily, there is a lot of literature about this topic, so we have decided to use a simplification of Liepa's algorithm [7], where new triangles are added inside the holes without adding new new vertices, and following a criterion of maximum equilaterality, similarly to what is done in the classical Delaunay triangulation. Other more complex and robust systems [8][9] could have been used but always being careful in the possible unwanted curvature added to the surface of the mesh.

\section{RESULTS}

The first substantial result obtained after the facial reconstruction process is a very noticeable increase of the polygonal resolution in the mesh, especially in the area belonging

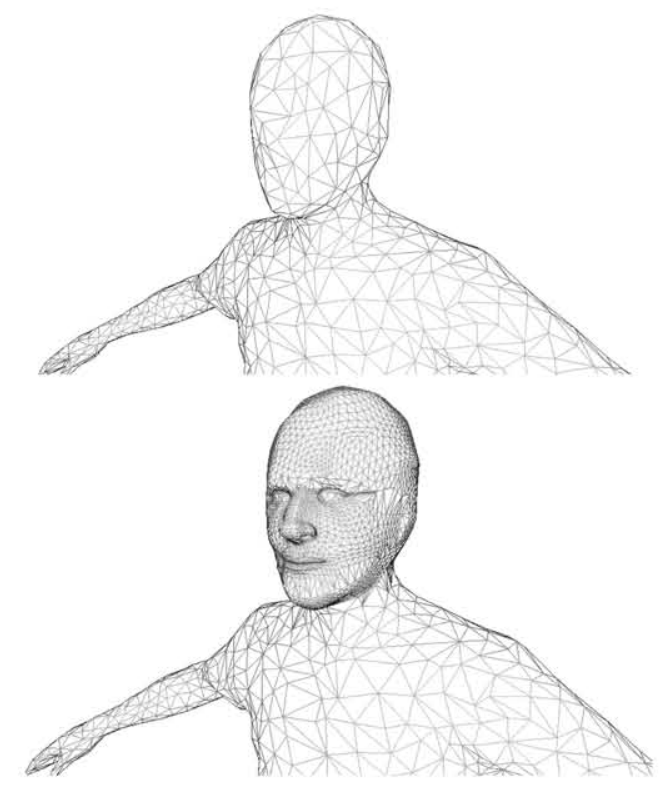

Figure 5. Original mesh and transformed mesh after the facial recontruction process, using the neutral face in Figure 1 (b).

to the head. This is mainly due to the triangle subdivision in this area and, furthermore, to the incorporation of the neutral reshaped mesh, which has the same density of triangles but, as it is more convoluted, has more surface. Visually, the results are very good (see Figures 5 and 6). The head area of the mesh is now very well defined. Facial features are easily distinguished, and the comparison of a mesh with the process applied vs. not applied is stunning. It is important to remark how good the results are using the same neutral mesh with two totally different people. We could even say that it is possible to recognise both subjects just by observing the mesh. If we had used more CPs (and, obviously, more TPs), the result would be even more similar to the reality.

One of the main advantages of our system is its geometrical simplicity but, as we can image, this could lead to visual artifacts when we finish filling the holes. The best way to avoid this is, again, having a big number of CPs, which will make the model more real, but it would be interesting to try to improve the last step of the hole filling process. Nevertheless, as we will see below, the results after texturing these meshes hide any possible irregularity in this process. A consequence of this simple geometrical nature of our system is that it does not need large computational resources, and it works well, even with complex meshes.

To show how accurate the system is when it represents human faces, we have applied a multi-view texturing mapping system [10] to both models and, as it is possible to see in Figure 6 (lowest image) and Figure 7, the result is very realistic. 

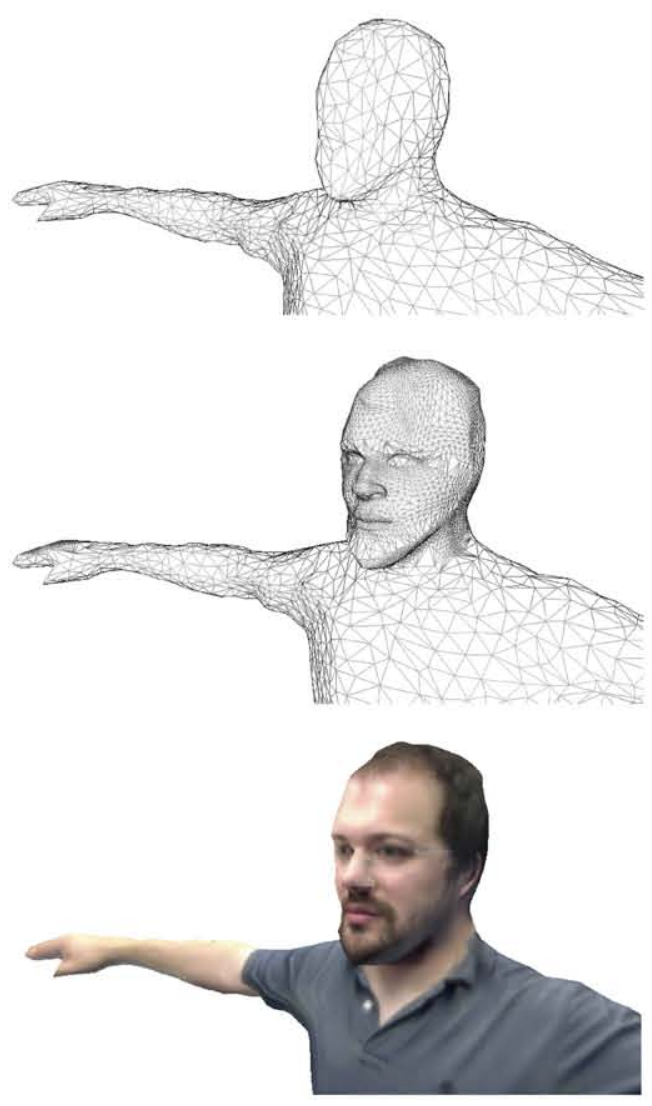

Figure 6. Original mesh, mesh after facial reconstruction and textured model.
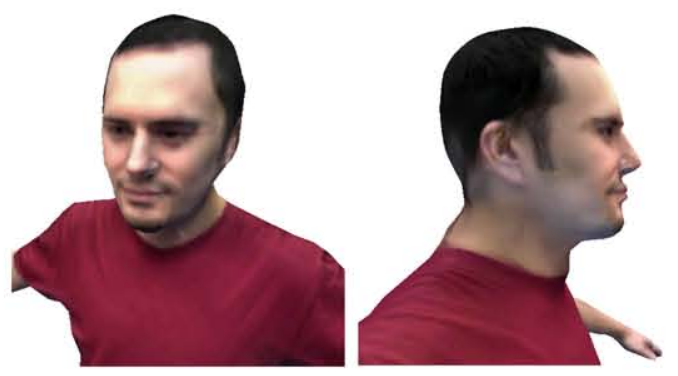

Figure 7. Two views of the model in Figure 1 after the texture is applied.

\section{CONCLUSIONS}

With the increasingly high demand of more realistic 3D models from video-game, film and many other industries, virtual human models must be more realistic, and the technology related to them as economic as possible. We have developed an innovative, efficient and easy-to-use system to reshape a generic neutral head mesh using the facial features of a particular person. Results show the good performance of the process which would probably be even better if we considered the use of different neutral meshes representing other sexes or races. Furthermore, as the CPs used in the process are known, it is possible to animate the resulting face, which is very interesting for using the models in different applications.

The main disadvantage of the process resides in its nonautomatic nature, so it would be interesting to research on automatic methods to obtain the CPs in the images. It is possible to detect these points using OpenCV if the image is a frontal photograph, but from a side-view photograph this is not so easy. Using an Intel Core Duo at $1,6 \mathrm{GHz}$ CPU with $2 \mathrm{~GB}$ of RAM, the process takes less than 15 seconds in average.

\section{ACKNOWLEDGMENTS}

This work has been partially supported by the Ministerio de Ciencia e Innovación of the Spanish Government under project TEC2010-20412 (Enhanced 3DTV). The authors also want to thank David Fuentes and Daniel and Berjón from GTI-UPM, and Mikel Fernández from Telefónica I+D in Barcelona for their invaluable cooperation.

\section{REFERENCES}

[1] D. Jiang, Y. Hu, S. Yan, L. Zhang, H. Zhang, and W. Gao, "Efficient 3D reconstruction for face recognition," Pattern Recognition, vol. 38, no. 6, pp. 787-798, 2005.

[2] N. Patel and M. Zaveri, "3D Facial Model Construction and Expressions Synthesis using a Single Frontal Face Image," International Journal on Graphics, vol. 1, 2010.

[3] W. Lee, P. Kalra, and N. Magnenat-Thalmann, "Model based face reconstruction for animation," Proc. Multimedia Modeling (MMM), pp. 323-338, 1997.

[4] V. Blanz and T. Vetter, "A morphable model for the synthesis of 3D faces," Proc. 26th Conf. on Computer graphics and interactive techniques, pp. 187-194, 1999.

[5] R. Hartley and A. Zisserman, Multiple view geometry. Cambridge University Press, Cambridge, UK, 2000.

[6] N. Dyn, D. Levine, and J. Gregory, "A butterfly subdivision scheme for surface interpolation with tension control," $A C M$ transactions on Graphics (TOG), vol. 9, no. 2, p. 169, 1990.

[7] P. Liepa, "Filling holes in meshes," Proc. 2003 EG/ACM symposium on Geometry processing, pp. 200-205, 2003.

[8] L. Tekumalla and E. Cohen, "A hole-filling algorithm for triangular meshes," School of Computing, University of Utah, UUCS-04-019, UT, USA, 2004.

[9] W. Zhao, S. Gao, and H. Lin, "A robust hole-filling algorithm for triangular mesh," The Visual Computer, vol. 23, no. 12, pp. 987-997, 2007.

[10] R. Pagés, S. Arnaldo, F. Morán, and D. Berjón, "Composition of texture atlases for 3D mesh multi-texturing," Proc. Eurographics-IT 2010 Conf., pp. 123-128, 2010. 